\title{
Sandblasting may damage the surface of composite CAD-CAM blocks
}

\author{
Kumiko Yoshihara ${ }^{a, *}$, Noriyuki Nagaoka ${ }^{b}$, Yukinori Maruo ${ }^{c}$, \\ Goro Nishigawa $^{c}$, Masao Irie ${ }^{d}$, Yasuhiro Yoshida ${ }^{e}$, Bart Van Meerbeek ${ }^{f}$ \\ a Centrer for Innovative Clinical Medicine, Okayama University Hospital, 2-5-1 Shikata-cho, Kita-ku, Okayama \\ 700-8558, Japan \\ b Advanced Research Center for Oral and Craniofacial Sciences, Okayama University Dental School, Okayama, Japan \\ c Department of Occlusion and Removable Prosthodontics, Okayama University Hospital, 2-5-1 Shikata-cho, \\ Kita-ku, Okayama 700-8558, Japan \\ d Department of Biomaterials, Okayama University Graduate School of Medicine, Dentistry and Pharmaceutical \\ Sciences, 2-5-1 Shikata-cho, Kita-ku, Okayama 700-8525, Japan \\ e Department of Biomaterials and Bioengineering, Graduate School of Dental Medicine, Hokkaido University, \\ Sapporo, Hokkaido, Japan \\ ${ }^{\mathrm{f}} \mathrm{KU}$ Leuven (University of Leuven), Department of Oral Health Sciences, BIOMAT \& University Hospitals Leuven, \\ Dentistry, Kapucijnenvoer 7, 3000 Leuven, Belgium
}

\section{A R T I C L E I N F O}

\section{Article history:}

Received 8 August 2016

Received in revised form

7 December 2016

Accepted 8 December 2016

Available online $\mathrm{xxx}$

Keywords:

composite

CAD-CAM

bond strength

sandblast

silane coupling agent

\begin{abstract}
A B S T R A C T
Objective. CAD-CAM blocks to fabricate semi-direct and indirect restorations are available in different sorts of ceramics as well as composite. In order to bond restorations prepared out of composite blocks into tooth cavities, it is recommended to gently sandblast the surface prior to the application of a primer/adhesive. Today, the effect of sandblasting composite block surfaces has not thoroughly been investigated. In this study, the ultra-structure of composite CAD-CAM blocks was investigated with special attention to the effect of sandblasting on the surface topography and of silanization on the bonding performance.

Methods. Five different composite CAD-CAM blocks were involved. We correlatively investigated their structural and chemical composition using X-ray diffraction (XRD), energy dispersion spectroscopy (EDS), scanning electron microscopy (SEM) and (scanning) transmission electron microscopy ((S)TEM). The effect of sandblasting was also imaged in cross-section and at the interface with composite cement. Finally, we measured the shear bond strength to the sandblasted block surface with and without silanization.

Results. All composite blocks revealed a different ultra-structure. Sandblasting increased surface roughness and resulted in an irregular surface with some filler exposure. Sandblasting also damaged the surface. When the sandblasted composite blocks were silanized, superior bonding receptiveness in terms of higher bond strength was achieved except for Shofu Block HC.

Significance. Sandblasting followed by silanization improved the bond strength to composite CAD-CAM blocks. However, sandblasting may also damage the composite CAD-CAM
\end{abstract}

\footnotetext{
* Corresponding author. Fax: +81 862356689.

E-mail address: k-yoshi@md.okayama-u.ac.jp (K. Yoshihara).

http://dx.doi.org/10.1016/j.dental.2016.12.003

0109-5641/@ 2016 The Academy of Dental Materials. Published by Elsevier Ltd. All rights reserved.
} 
block surface. For the composite CAD-CAM block Shofu Block HC, the damage was so severe that silanization did not improve bond strength.

๑ 2016 The Academy of Dental Materials. Published by Elsevier Ltd. All rights reserved.

\section{Introduction}

In recent years, the fast progress in computer-assisted technology has dramatically changed today's dental practice [1]. Chairside CAD-CAM technology has already been available for more than 25 years in the form of the first-generation Cerec (Sirona, Bensheim, Germany) system. This technology has evolved to a well-established system, enabling either to construct semi-direct partial- or full-crown restorations or to apply it for digital impressioning, so the fixed partial denture (FPD) can be designed digitally and eventually milled in the dental technician lab [2]. Today, other in-office CAD-CAM systems, like the E4D system (Planmeca, Helsinki, Finland), are available as well as there currently exists a plethora of intraoral scanners for digital impressioning; regarding the latter, one may expect that the use of conventional impressioning techniques using elastomeric impression materials will decrease in favor of digital impressioning.

Along with the fast innovation in digital dental devices, new CAD-CAM blocks in different sorts of ceramics have been developed, while most recently also CAD-CAM blocks in composite or in a ceramic-composite mixture, often being referred to as 'polymer-infiltrated' or 'hybrid' ceramic, have been produced [3]. One of the first composite CAD-CAM blocks was Paradigm MZ100 (3M ESPE, St. Paul, MN, USA), but was only commercially available in certain markets [4]. Since then, other composite CAD-CAM blocks were developed, using novel techniques to reach better degrees of conversion or more favorable filler loading and distributions [5]. One of the major advantages of composite CAD-CAM blocks is the better milling accuracy; composite chips less at the restoration margin during milling than ceramic does [4]. In addition, composite is more gentle in terms of abrasion for the opposing teeth [6]. Some papers also documented that composite CAD-CAM blocks are superior in fatigue or fracture resistance than glass ceramics $[7,8]$. Due to a recent installment of health insurance reimbursement in Japan regarding the use of composite CAD-CAM blocks for restoring premolars, many Japanese dental manufacturers have launched new composite CAD-CAM blocks to the Japanese market, of which some are also available outside Japan. Also noteworthy is the documented high de-bonding rate of Lava Ultimate (3M ESPE) composite blocks, having forced 3M ESPE to no longer support the indication to fabricate solitary crowns $[9,10]$.

Since composite CAD-CAM blocks are relatively new materials, laboratory and clinical data are highly needed. Therefore, the structure of five composite CAD-CAM blocks was investigated, in particular to assess (1) the effect of sandblasting on their surface topography and (2) the effect of sandblasting and silanization on their bonding receptiveness. The null hypothesis investigated was that composite CAD-CAM blocks do not benefit from sandblasting and silanization with regard to their bonding receptiveness.

\section{Materials and methods}

Five composite CAD-CAM blocks were investigated in this study (Table 1).

\subsection{Scanning electron microscopy (SEM) of untreated composite CAD-CAM blocks}

From block size 14, thin 1-mm thick slices were cut using a semi-automated high-speed diamond saw (Accutom, Struers, Ballerup, Denmark). For each brand, three CAD-CAM blocks were cross-sectioned by argon-ion milling (SM-090101 CrossSection Polisher, JEOL, Tokyo, Japan). Subsequently, a thin layer of carbon was vaporized on the surface (JEE-420T Vacuum Evaporators, JEOL), after which the specimens were examined using Field-emission-gun SEM (Feg-SEM; JSM-6701F, JEOL), being operated at $5 \mathrm{kV}$ and using an annular semi-conductor detector.

\subsection{Scanning transmission electron microscopy (STEM) of untreated composite CAD-CAM blocks}

Following SEM, the cross-sections were further processed for STEM using an argon-ion slicer (EM-09100IS Ion Slicer, JEOL). STEM (JEM-2100F, JEOL) was carried out employing an accelerating voltage of $200 \mathrm{kV}$ and current density of $40 \mathrm{pA} / \mathrm{cm}^{2}$. Again, three different specimens for each composite CAD-CAM block were examined.

\subsection{X-ray diffraction (XRD) of untreated composite CAD-CAM blocks}

From block size 14, thin 1-mm thick slices were cut using the semi-automated high-speed diamond saw (Accutom, Struers). The surface was ground and polished using a $15-\mu \mathrm{m}$ diamond lapping film in order to reach a mirror-polished surface. Three different specimens for each composite CAD-CAM block were prepared.

The crystal phases of the specimens were identified using an X-ray powder diffractometer (CuK $\alpha 1$ 1.5406 ̊, RINT 2500, Rigaku, Tokyo, Japan), operated at $40 \mathrm{kV}$ acceleration and $200 \mathrm{~mA}$ current and a scanning rate of $0.02^{\circ} \mathrm{s}^{-1}$ for $2 \theta / \theta$ scans.

\subsection{SEM of sandblasted composite CAD-CAM blocks}

Using the methods described above, additional 1-mm thick and mirror-polished specimens were prepared and sandblasted using a laboratory sandblaster (Hi-Blaster III, Shofu, Kyoto, Japan). With 50- $\mu \mathrm{m}$ aluminium oxide (HI ALUMINAS, 


\begin{tabular}{|c|c|c|c|c|c|}
\hline Product name & Company & Monomer composition & Filler composition & $\begin{array}{l}\text { Filler } \\
\text { mass } \\
\text { (wt\%) }\end{array}$ & $\begin{array}{l}\text { Flexural } \\
\text { strength } \\
\text { (MPa) }\end{array}$ \\
\hline Cerasmart & GC, Tokyo, Japan & $\begin{array}{l}\text { Bis-MEPP, UDMA, } \\
\text { DMA }\end{array}$ & $\begin{array}{l}\mathrm{SiO}_{2}(20 \mathrm{~nm}) \text {, barium } \\
\text { glass }(300 \mathrm{~nm})\end{array}$ & 71 & 240 \\
\hline Katana Avencia & Kuraray Noritake, Tokyo, Japan & $\begin{array}{l}\text { UDMA, methacrylate } \\
\text { monomer }\end{array}$ & $\begin{array}{l}\mathrm{SiO}_{2}(40 \mathrm{~nm}), \mathrm{Al}_{2} \mathrm{O}_{3} \\
(20 \mathrm{~nm})\end{array}$ & 62 & 231 \\
\hline KZR-CAD HR & Yamakin, Osaka, Japan & UDMA, TEGDMA & $\begin{array}{l}\mathrm{SiO}_{2}-\mathrm{Al}_{2} \mathrm{O}_{3}-\mathrm{ZrO}_{2} \\
(200-600 \mathrm{~nm}), \mathrm{SiO}_{2} \\
(20 \mathrm{~nm}, 100 \mathrm{~nm})\end{array}$ & 73 & 235 \\
\hline Lava Ultimate & 3M ESPE, St. Paul, MN, USA & $\begin{array}{l}\text { Bis-GMA, UDMA, } \\
\text { bis-EMA, TEGDMA }\end{array}$ & $\begin{array}{l}\mathrm{SiO}_{2}(20 \mathrm{~nm}), \mathrm{ZrO}_{2} \\
\text { (4-11 nm), } \mathrm{ZrO}_{2} / \mathrm{SiO}_{2} \\
\text { clusters }\end{array}$ & 80 & 204 \\
\hline Shofu Block HC & Shofu, Kyoto, Japan & UDMA, TEGDMA & $\mathrm{SiO}_{2}$, zirconium silicate & 61 & 191 \\
\hline
\end{tabular}

Shofu, Kyoto, Japan) and at an air pressure of $0.2 \mathrm{MPa}$ for $10 \mathrm{~s}$, the device was held at a distance of $1 \mathrm{~cm}$ from the target surface. After coating the sandblasted surface with a thin layer of osmium (Neo Osmium Coater, Meiwafosis, Tokyo, Japan), FegSEM equipped with in-lens detectors (JSM-6701F, JEOL) was used to acquire high-resolution photomicrographs.

For SEM of the cross-sections, additional slice specimens were subjected to argon-ion milling (SM-090101 Cross-Section Polisher, JEOL) and osmium coating prior to Feg-SEM analysis. Three different specimens for each composite CAD-CAM block were examined.

\subsection{Chemical elemental analysis of sandblasted composite CAD-CAM blocks}

Element distributions of the sandblasted composite CAD-CAM blocks were determined using SEM (DC-720, Topcon, Tokyo, Japan) equipped with Energy dispersive X-ray spectroscopy (EDS; Noran Voyager III M3100, Noran Instruments, Middleton, Wisconsin, USA). Three different specimens for each composite CAD-CAM block were analyzed.

\subsection{Shear bond strength (SBS) to sandblasted composite CAD-CAM blocks}

Twenty $10 \times 10 \times 1-\mathrm{mm}$ composite specimens were sandblasted as described above. Ten specimens were kept as such, while the second set of 10 specimens were treated with a silane-containing ceramic primer before cementation.

Zirconia cylinders were used to measure the bond strength of cement to the composite CAD-CAM blocks; Zirconia is strong enough and a composite cement can strongly be bonded to zirconia on the condition that it beforehand is sandblasted and chemically pre-treated with a combined MDP (10-methacryloyloxydecyl dihydrogen phosphate)/silane ceramic primer [11]. Zirconia (Tosoh, Tokyo, Japan) cylinders of 2-mm height and 3.6- $\mathrm{mm}$ diameter were therefore sandblasted using a laboratory sandblaster (Hi-Blaster III, Shofu) with $50-\mu \mathrm{m}$ aluminium oxide (Shofu) and at an air pressure of $4.0 \mathrm{MPa}$ for $10 \mathrm{~s}$. The device was held at a distance of $1 \mathrm{~cm}$ from the target zirconia surface. After having been treated with an MDP/silane-containing ceramic primer (Clearfil Ceramic Primer, Kuraray Noritake, Tokyo, Japan), the zirconia cylinders were luted to the composite CAD-CAM block specimens using a composite cement (Clearfil Esthetic Cement, Kuraray Noritake).

All bonded specimens were light-cured for $40 \mathrm{~s}$ from two opposite directions (totaling to a final curing time of $80 \mathrm{~s}$ ) using G-Light Prima II Plus (light intensity of $2800 \mathrm{~mW} / \mathrm{cm}^{2}$; GC, Tokyo, Japan). After curing, the bonded specimens were stored in distilled water at $37^{\circ} \mathrm{C}$ for $24 \mathrm{~h}$. To measure the shear-bond strength, the bonded specimens were mounted in a universal testing machine (Model 5565, Instron, Canton, MA, USA) and exposed to shear stress at a cross-head speed of $0.5 \mathrm{~mm} / \mathrm{min}$. All fractured specimens were analyzed with a light microscope $(4 \times)$ (SMZ-10, Nikon, Tokyo, Japan) to determine the fracture mode.

For statistical comparison, two-way ANOVA followed by Tukey's multiple comparison test was used, whereby $\mathrm{p}<0.05$ was considered statistically significant.

\subsection{Cross-section SEM and STEM of cement-composite CAD-CAM block interfaces}

Each composite CAD-CAM block was sandblasted using a laboratory sandblaster (Hi-Blaster III, Shofu) with $50-\mu \mathrm{m}$ aluminium oxide at $0.2-\mathrm{MPa}$ air pressure. All sandblasted surfaces were treated with Clearfil Ceramic primer (Kuraray Noritake), upon which a luting composite (Clearfil Esthetic Cement, Kuraray Noritake) was applied and light-cured for 40 s (G-Light Prima II Plus). Cross-section specimens were prepared as described above and then observed using SEM and STEM.

\section{Results}

\subsection{SEM of untreated composite CAD-CAM blocks (Fig. 1)}

Different filler configurations were observed for the five composite CAD-CAM blocks investigated. The CAD-CAM blocks Cerasmart (GC) and in particular Katana Avencia (Kuraray Noritake) contained the smallest filler particles at least at low magnification (Fig. 1a,c). High magnification of Cerasmart revealed a filler size of maximum $1 \mu \mathrm{m}$ 


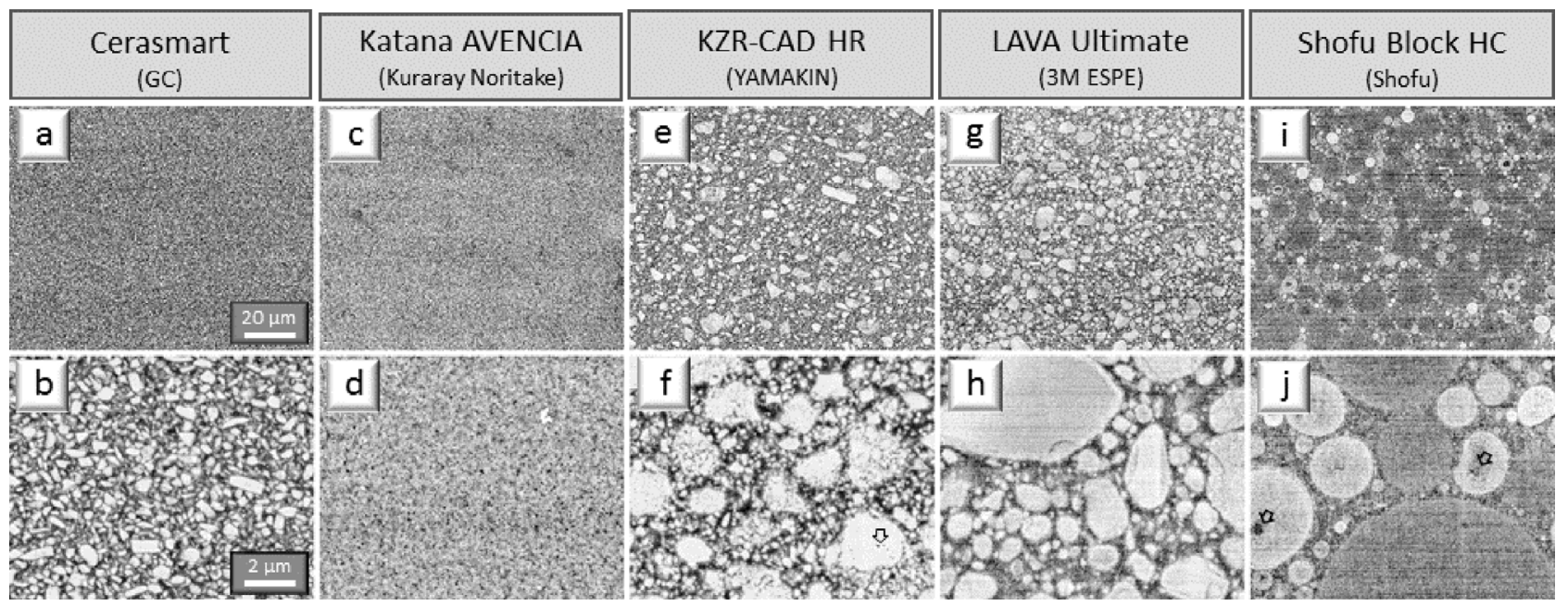

Fig. 1 - SEM photomicrographs of untreated composite CAD-CAM blocks. (a,b) The CAD-CAM blocks Cerasmart (GC) contained the smallest filler particles at least at low magnification (a). High magnification of Cerasmart revealed a filler size of maximum $1 \mu \mathrm{m}$ (b). (c,d) Katana Avencia (Kuraray Noritake) showed varying nanometer-sized filler particle sizes ranging between 20 and $50 \mathrm{~nm}$ (e,f) KZR-CAD HR (Yamakin) presented with different filler-particle sizes ranging between 1 and $10 \mu \mathrm{m}$ (e). The large filler particles were constituted of agglomerated small filler particles. A clear void was observed in a large filler cluster (f: arrows). (g,h) Lava Ultimate (3M ESPE) presented with relatively large filler particles of 5-10 $\mu \mathrm{m}$ as well as much small filler particles. (i,j) Shofu Block HC (Shofu) typically contained spherical filler particles ranging between 1 and $10 \mu \mathrm{m}$ (i). High magnification of Shofu Block HC revealed holes within the filler particles (j: arrows).

(Fig. 1b). High-magnification of Katana Avencia showed varying nanometer-sized filler particle sizes ranging between 20 and $50 \mathrm{~nm}$ (Fig. 1d).

KZR-CAD HR (Yamakin) presented with different fillerparticle sizes ranging between 1 and $10 \mu \mathrm{m}$ (Fig. 1e). The large filler particles were constituted of agglomerated small filler particles. A clear void was observed in a large filler cluster, where was not penetrated by resin (Fig. If arrow). Lava Ultimate (3M ESPE) presented with relatively large filler particles of 5-10 $\mu \mathrm{m}$ as well as much small filler particles (Fig. 1g,h). Shofu Block HC (Shofu) typically contained spherical filler particles ranging between 1 and $10 \mu \mathrm{m}$ (Fig. 1i,J). High magnification of Shofu Block HC revealed holes within the filler particles (Fig. 1j arrow).

\subsection{STEM observation of untreated composite CAD-CAM blocks (Fig. 2)}

STEM imaging provided more ultrastructural details of each CAD-CAM block. High magnification of Cerasmart revealed 10-30 nm filler particles interspersed between large angular filler particles (Fig. 2a-c). Katana Avencia showed a very high filler density (Fig. 2d-f) with the primary filler size ranging between 20 and $100 \mathrm{~nm}$ (Fig. 2f). KZE-CAD HR typically revealed filler clusters (Fig. 2g-i). Small filler particles of $10-30 \mathrm{~nm}$ were abundantly interspersed between the large filler particles (Fig. 2i). Lava Ultimate also contained filler clusters. They were constituted of larger core fillers of $1-3 \mu \mathrm{m}$ (Fig. $2 \mathrm{j}-1$ ) and small spherical fillers, some of which were less than $10 \mathrm{~nm}$ (Fig. 21). Shofu Block HC showed relatively large spherical filler particles with peculiar holes (Fig. $2 \mathrm{~m}, \mathrm{n}$ ). The holes, in turn, were filled with small filler particles (Fig. 2m,n). In addition, small nanometer-sized filler particles were also abundantly interspersed between the larger spherical fillers (Fig. 2n,o).

\subsection{XRD of untreated composite CAD-CAM blocks (Fig. 3)}

All composite CAD-CAM blocks showed a broad peak around $20^{\circ}$ which most likely must be assigned to an amorphous phase. The CAD-CAM blocks KZR-CAD HR and Lava Ultimate additionally showed four characteristic peaks, which should be assigned to $\mathrm{ZrO}_{2}$ (arrows in Fig. 3).

\subsection{SEM of sandblasted composite CAD-CAM blocks (Figs. 4 and 5)}

Feg-SEM photomicrographs of all sandblasted CAD-CAM blocks revealed irregular and rough surfaces (Fig. 4), except for KZR-CAD that remained relatively flat after sandblasting (Fig. 4g-i). Characteristics were the spherical holes detected at the surface of the sandblasted Shofu Block HC (Fig. 4m). Highmagnification of the sandblasted Shofu Block $\mathrm{HC}$ revealed a smooth surface within the spherical holes (Fig. 4n).

The cross-sections clearly revealed the irregular surface sandblasting produced at all CAD-CAM blocks (Fig. 5). In Cerasmart and Katana Avencia, only a rather small crack was detected close to the sandblasted surface (Fig. 5b,c and e,f, respectively), while the latter block also presented a local spot of deeper block damage (Fig. 5e,f). The sandblasted surface of the KZR-CAD HR block was clearly more severely damaged; it showed several cracks, of which some ended at the surface, while also some sub-surface cracks deeper in the block could be detected (Fig. 5i). High magnification photomicrographs of KZR-CAD HR and Lava Ultimate (Fig. 5h,i and k,l, respectively) 


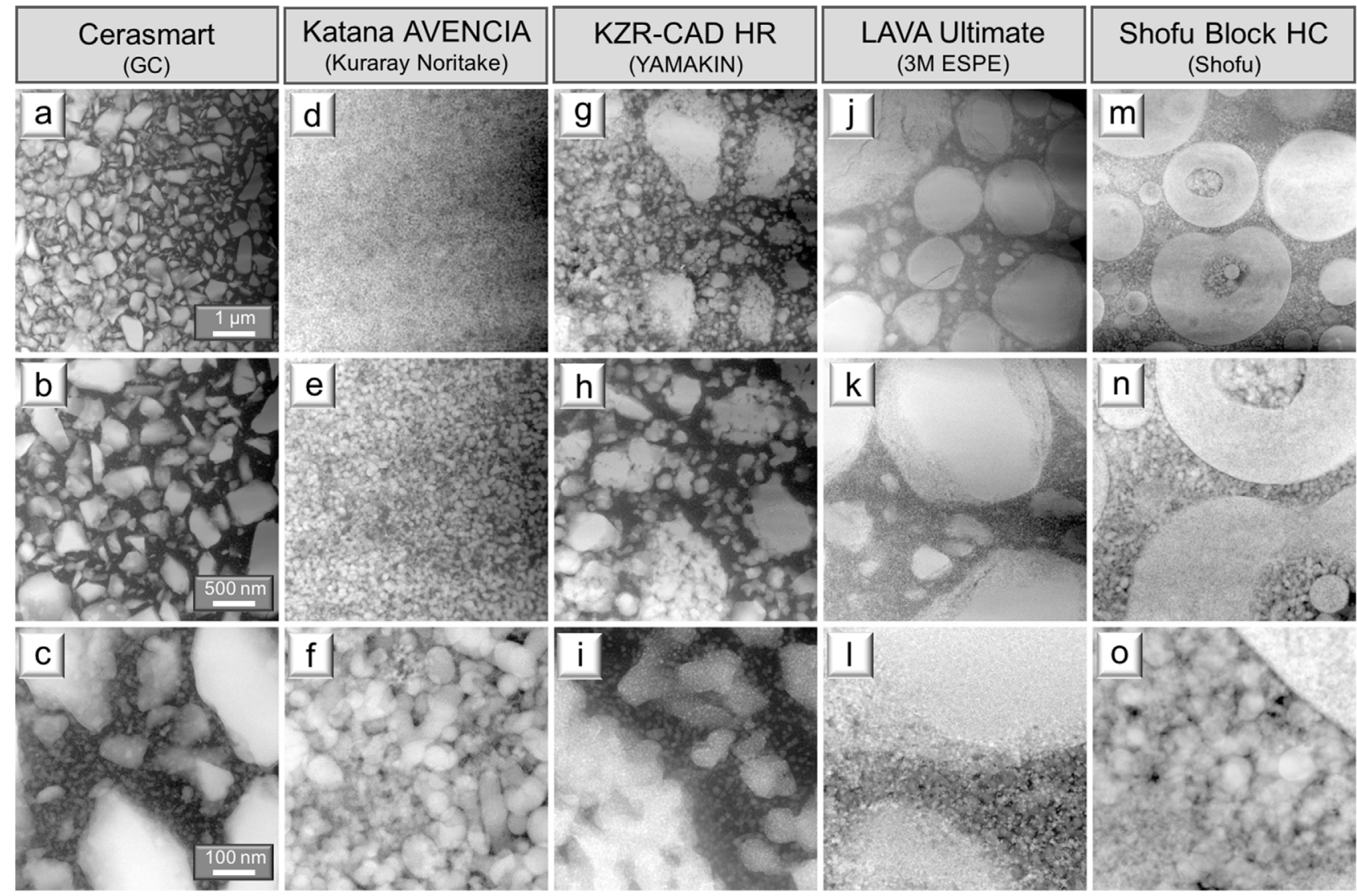

Fig. 2 - STEM photomicrographs of untreated composite CAD-CAM blocks. (a-c) High magnification of Cerasmart revealed 10-30 nm filler particles interspersed between large angular filler particles $(b, c)$. (d-f) Katana Avencia showed a very high filler density (d-f), with the primary filler size ranging between 20 and $100 \mathrm{~nm}$ (f) (g-i) KZE-CAD HR typically revealed filler clusters (g-i). Small filler particles of $10-30 \mathrm{~nm}$ were abundantly interspersed between the large filler particles (i). (j-l) Lava Ultimate also contained filler clusters. They were constituted of larger core fillers of $1-3 \mu \mathrm{m}(j-1)$ and small spherical fillers, some of which were less than $10 \mathrm{~nm}(\mathrm{l})$. $(\mathrm{m}-\mathrm{o})$ Shofu Block HC showed relatively large spherical filler particles with peculiar holes $(m, n)$. The holes, in turn, were filled with small filler particles $(m, n)$. In addition, small nanometer-sized filler particles were also abundantly interspersed between the larger spherical fillers $(n, 0)$.

revealed several cracks within the large filler particles as well as resin matrix. Most severe damage was observed at the sandblasted surface of Shofu Block HC; one very large crack was shown to have propagated along several filler particles (Fig. 5m-o).

\subsection{Chemical element distribution maps of} sandblasted composite CAD-CAM blocks (Fig. 6)

The elements carbon $(\mathrm{C})$, oxygen $(\mathrm{O})$, silicon (Si), and aluminum (Al) were detected in all composite CAD-CAM blocks. In KZR-CAD HR, Lava Ultimate and Shofu Block HC, zirconium (Zr) was detected as well. Only in Cerasmart, barium (Ba) was found to be rather homogenously spread. In Cerasmart, LAVA Ultimate and Katana Avencia, Al was identified.

\subsection{Shear bond strength (SBS) to sandblasted} composite CAD-CAM blocks (Fig. 7)

All sandblasted composite CAD-CAM blocks demonstrated a statistically similar SBS. Silane treatment after sandblasting led to a significantly higher SBS for all blocks, except for Shofu Block HC.

\subsection{Cross-section SEM and STEM of cement-composite CAD-CAM block interfaces (Figs. 8 and 9)}

Except for the CAD-CAM block Shofu Block HC, a tight interface of the composite cement to all composite CAD-CAM blocks was revealed by SEM (Fig. 8); the cement could infiltrate into the roughened sandblasted surface of the composite blocks. However, not all sub-surface cracks were infiltrated by the cement (arrows in Fig. 8c,f,l,o). In particular for Shofu Block HC, several voids could be observed along the cement-block interface (Fig. 8n,o). Further, the cement could not infiltrate into the cracks that had propagated sub-surfacely along the filler particles.

STEM confirmed the SEM findings, also revealing a tight cement-block interface (Fig. 9). In Shofu Block HC, clear cracks that had propagated along the spherical fillers could be observed (Fig. 9m,n). 


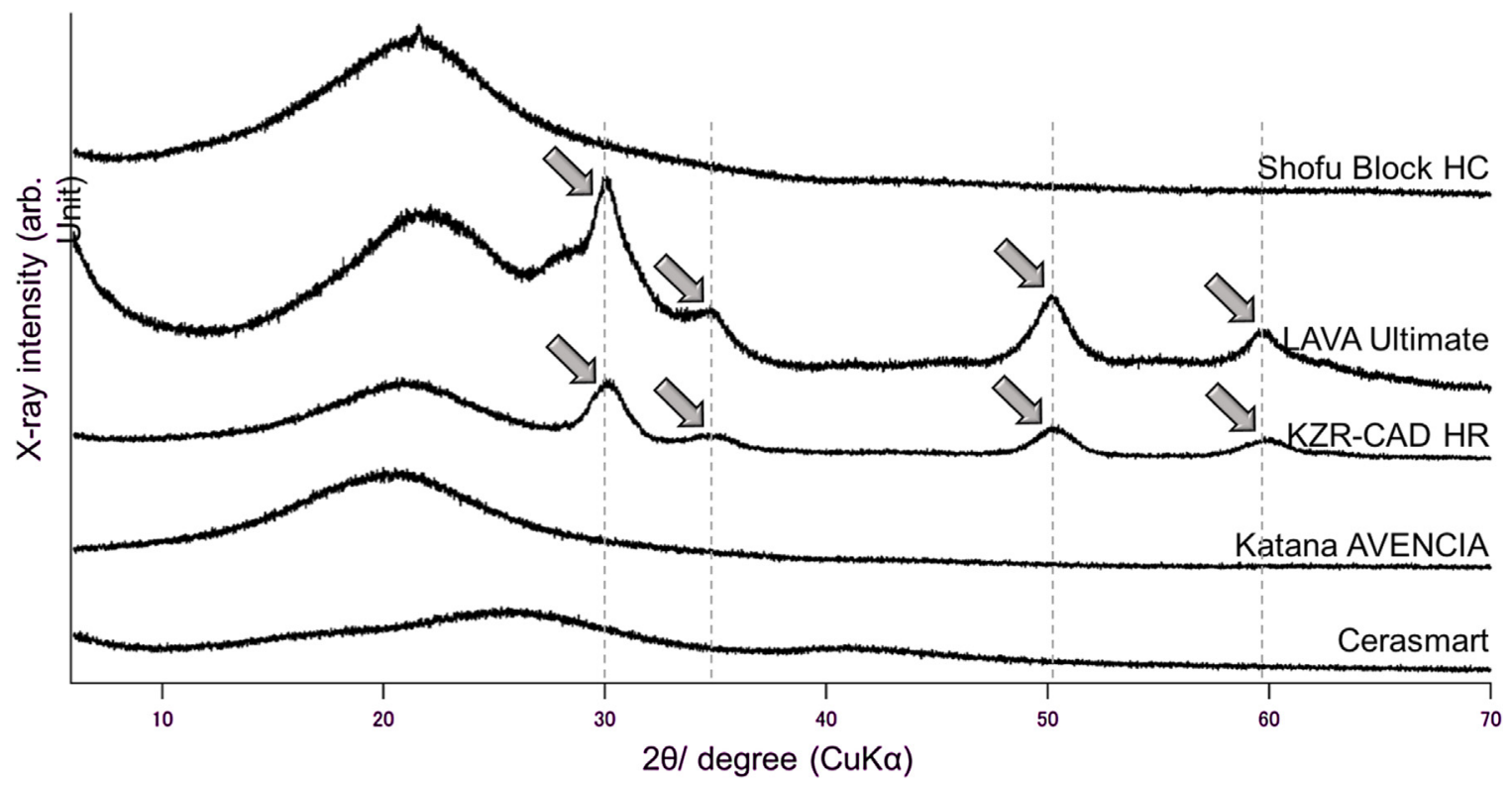

Fig. 3 - XRD patterns of the composite CAD-CAM blocks studied. All composite CAD-CAM blocks showed a broad peak around $20^{\circ}$, which most likely must be assigned to an amorphous phase. The CAD-CAM blocks KZR-CAD HR and Lava Ultimate additionally showed four characteristic peaks, which should be assigned to $\mathrm{ZrO}_{2}$ (arrows).

\section{Discussion}

In daily dental practice, sandblasting is a common procedure applied to clean and increase the surface energy of the internal side of ceramic (and metal) crown restorations, most commonly with the intention to improve bonding receptiveness. Sandblasting was indeed shown to improve bond strength by exposing a fresh surface, free of contaminants, and by providing enhanced micro-mechanical retention to the cement at the roughened surface [12-14]. Also to adhesively

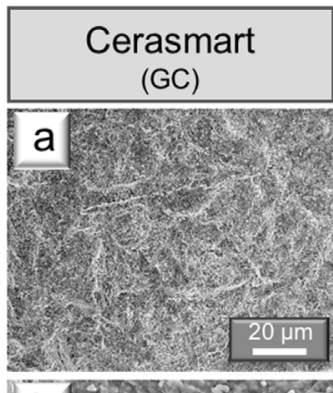

Katana AVENCIA
(Kuraray Noritake)

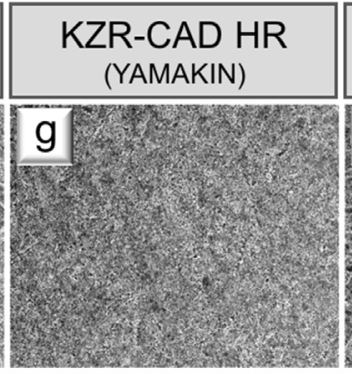

LAVA Ultimate
(3M ESPE)
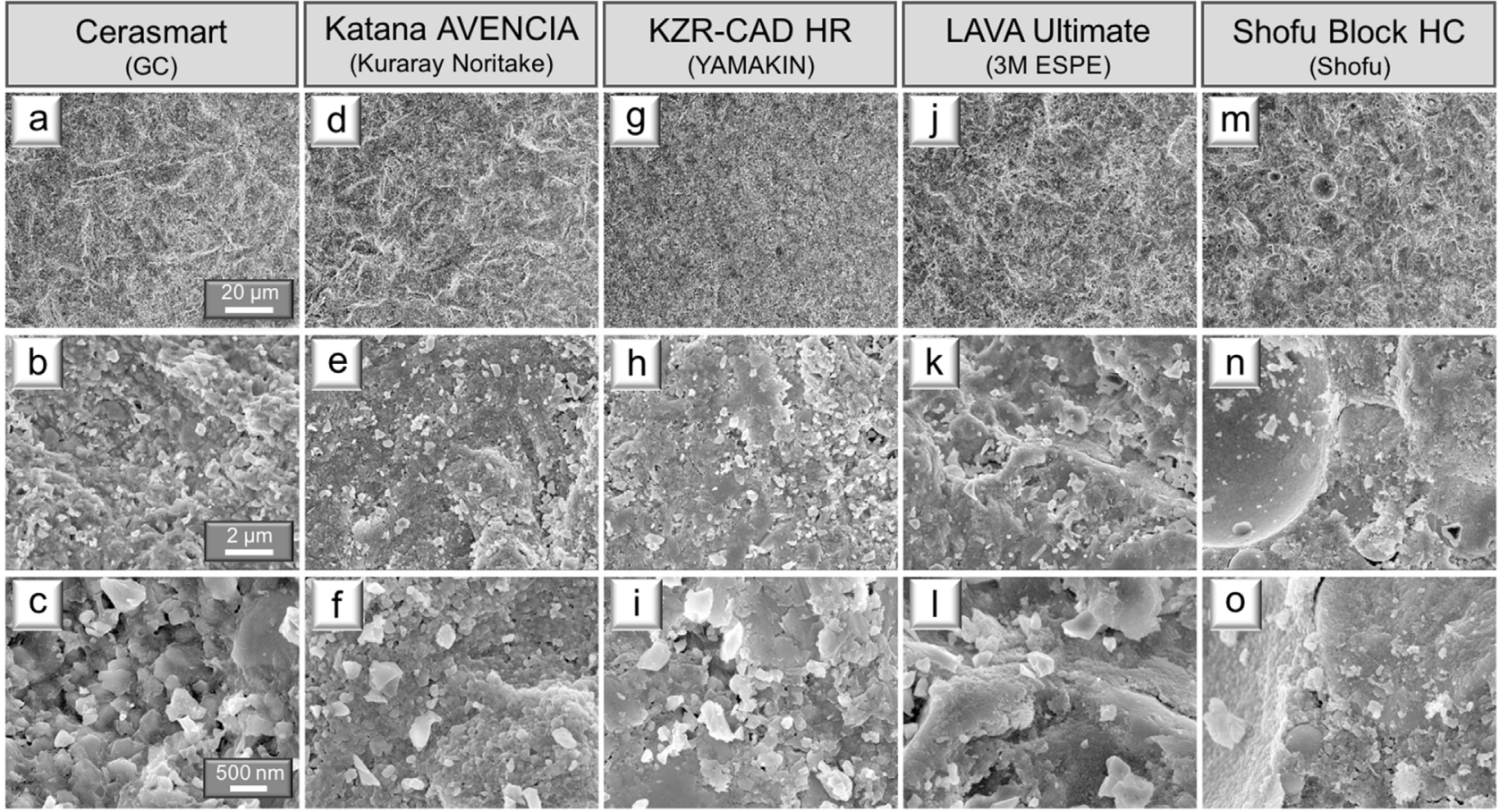

Fig. 4 - Feg-SEM photomicrographs of the sandblasted surfaces of the composite CAD-CAM blocks. (a-c) Cerasmart; (d-f) Katana Avencia; (g-i) KZR-CAD HR; (j-l) Lava Ultimate; (m-o) Shofu Block HC. All CAD-CAM blocks showed an irregular and rough surface, except for KZR-CAD HR that remained relatively flat (g,h). Only Shofu Block HC showed spherical holes (m) and a smooth surface within the holes (n). 


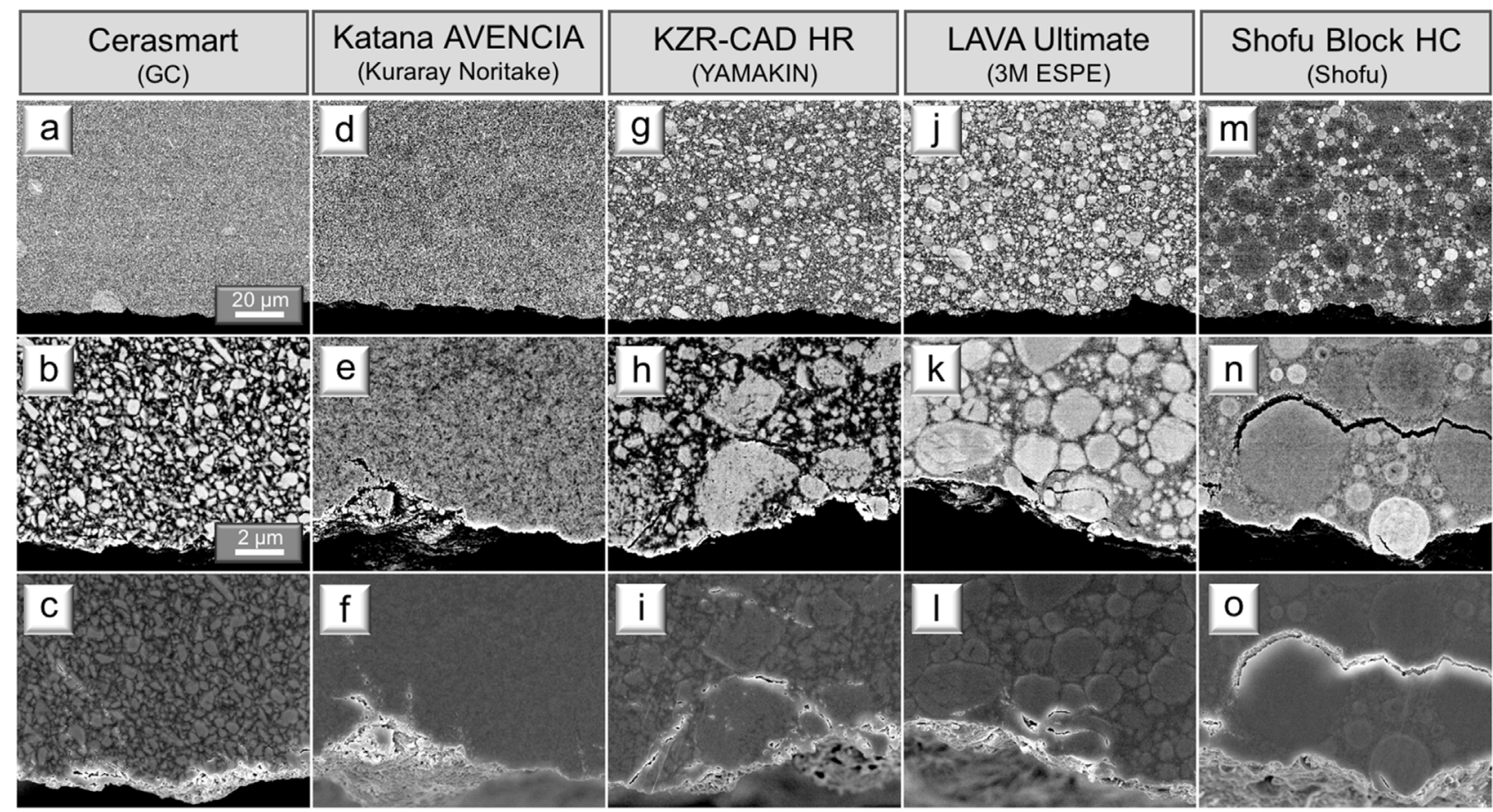

Fig. 5 - Feg-SEM photomicrographs of the sandblasted composite CAD-CAM blocks in cross-section. (a-c) Cerasmart; (d-f) Katana Avencia; (g-i) KZR-CAD HR; (j-l) Lava Ultimate; $(m-0)$ Shofu Block HC. All CAD-CAM blocks revealed an irregular surface upon sandblasting $(a, d, g, j, m)$. Cerasmart showed a small crack near the sandblasted surface $(b, c)$. The sandblasted surface of Katana Avencia was locally more severely damaged with one crack running deeper in the block (e,f). The sandblasted surface of the KZR-CAD HR block was clearly more severely damaged; it showed several cracks, of which some ended at the surface, while also some sub-surface cracks deeper in the block could be detected (h,i). High magnification of Lava Ultimate showed cracks within the large filler particles and resin matrix (k,l). Shofu Block HC revealed the largest damage with large cracks that had propagated along several filler particles (m-o).

repair defective existing composite restorations, sandblasting is highly recommended [12,15-18].

The advancement of dental CAD-CAM technology has triggered dental industry to produce materials in the form of blocks that can be processed by CAD-CAM in the dental technician laboratory (or milling centers) as part of an 'indirect' restorative technique, but also to be processed chairside in the dental cabinet as part of a 'semi-direct', one-visit restorative technique. A new generation of CAD-CAM blocks is made of resin composite. Compared with dental composite intended for 'direct' intra-oral use, the mechanical properties of composite CAD-CAM blocks are significantly better because these blocks can be industrially polymerized at higher temperature and pressure [4]. Nonetheless, the basic composition of these composite blocks is quite alike that of 'direct' composites delivered in pastes (Table 1), by which one could expect that they can be adhesively luted (bonded) using similar adhesive systems and approaches. Obviously, the high degree of polymerization of the composite blocks necessitates to adequately pre-treat the block surface for bonding purposes. Although the bonding receptiveness of some composite blocks can also be improved by etching with hydrofluoric acid $[19,20]$, most composite CAD-CAM blocks are recommended to be sandblasted prior to silanization [21-25]. However, the impact sandblasting can have on the surface integrity of the composite blocks is today insufficiently researched, as well as the effect of silanization of composite CAD-CAM blocks on bonding performance deserved more study.

The five different composite CAD-CAM blocks investigated in this study contained $61-80 \mathrm{wt} \%$ filler (Table 1). SEM and STEM analyses revealed widely varying filler-matrix ultrastructures. Cerasmart mainly contained irregularly shaped filler of maximum $1 \mu \mathrm{m}$; STEM further revealed the presence of 10-30 nm filler particles, which could not be observed by SEM. Katana Avencia revealed a nanometer-sized filler configuration with particles ranging between 20 and $50 \mathrm{~nm}$. According to Katana Avencia's manufacturer, this block was fabricated by pressing filler followed by monomer infiltration [5], thereby obtaining composite CAD-CAM blocks with nano-sized filler [5]. In contrast, KZR-CAD HR, Lava Ultimate and Shofu Block HC contained relatively large filler as well as nano-filler. In KZR-CAD HR, the large filler particles were constituted of clusters of small filler; voids and spaces could be detected within the filler clusters. Lava Ultimate consisted of filler particles differing in size and composition. Shofu Block HC characteristically showed spherical filler particles, some of which exhibiting holes.

XRD analysis revealed that KZR-CAD HR and Lava Ultimate contained crystalline $\mathrm{ZrO}_{2}$, whereas the other blocks contained non-crystalline resin and glass. EDS mapping revealed that also Shofu Block HC contained zirconium in the form of zirconium metallic glass [10]. Obviously, these differences in 


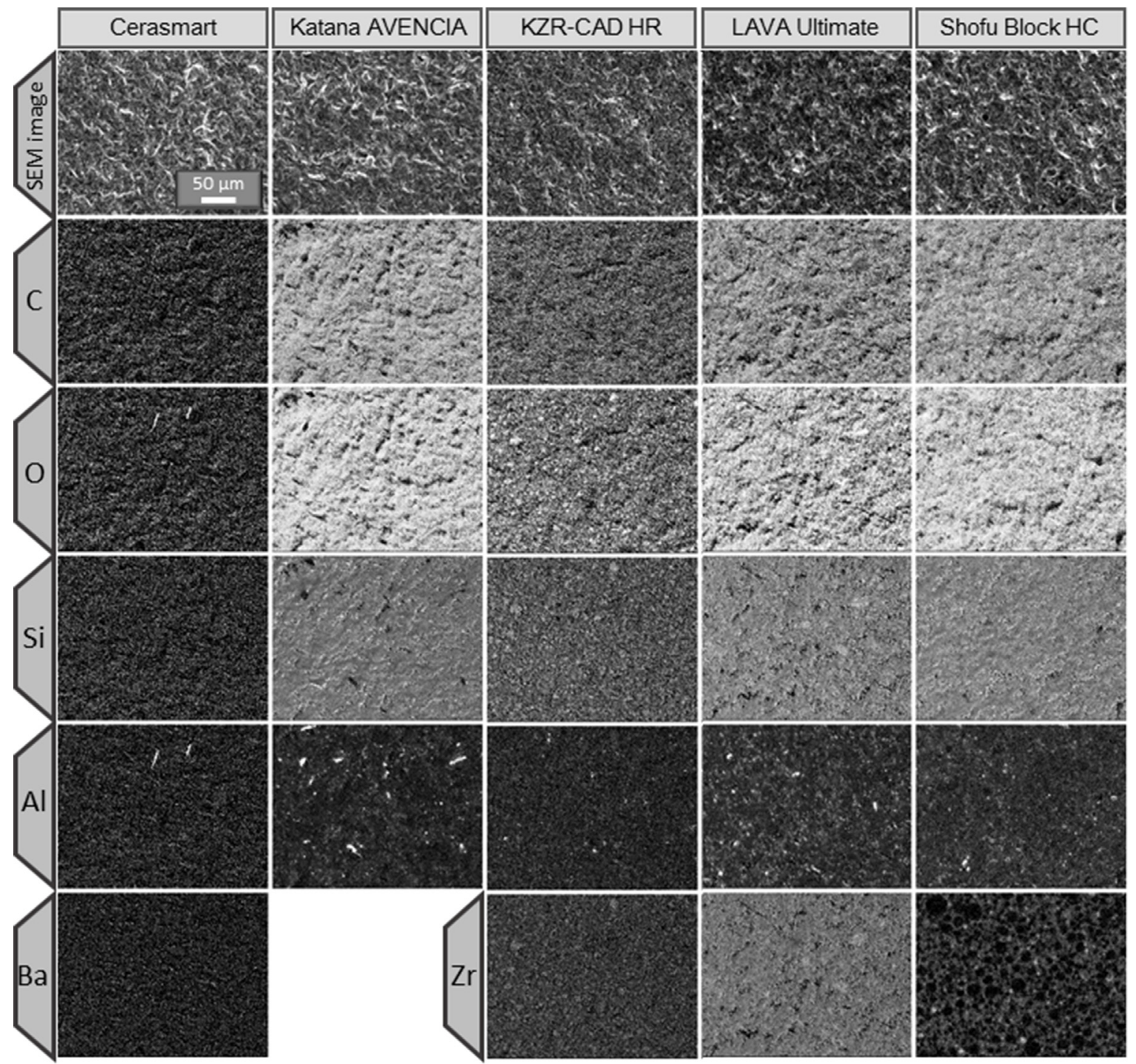

Fig. 6 - SEM/EDS mapping of the sandblasted composite CAD-CAM blocks. Carbon (C), oxygen (O), silicon (Si) and aluminum (Al) were detected in all CAD-CAM blocks. Barium $(\mathrm{Ba})$ was detected in Shofu Block HC only; zirconium ( $\mathrm{Zr}$ ) was detected in KZR-CAD HR, Lava Ultimate and Shofu Block HC. Al was detected more intensively in localized spots for Cerasmart, LAVA Ultimate and Katana Avencia.

filler-matrix configuration/composition among the composite CAD-CAM blocks must also be expressed in different mechanical properties for these blocks.

In order to adhesively lute composite CAD-CAM blocks, sandblasting is commonly recommended [21-25]. The recommended sandblasting pressure is $0.1-0.2 \mathrm{MPa}$, which is lower than the pressure commonly recommended for ceramic and metal restorations. Such reduced pressure is desired because composite CAD-CAM blocks have a lower Vickers hardness than (glass-)ceramics [4]. In this study, the surfaces were sandblasted at 0.2 MPa. We experimented with 0.1-MPa pressure, but this appeared insufficient to deliver uniformly sandblasted surfaces (data not shown).

SEM of the sandblasted block surfaces revealed an irregular surface for all the composite CAD-CAM blocks investigated in this study. Cross-section imaging confirmed that the sandblasted surfaces were not flat. Some parts were deeply dented, most likely because these local spots were directly hit by one or more impacting alumina particles used for sandblasting. SEM-EDS revealed that some alumina particles remained attached onto the surface. Sandblasting also caused cracks of $1-10 \mu \mathrm{m}$ length at the surfaces of all the composite CAD-CAM blocks, as it was particularly demonstrated by SEM in the high-magnification cross-section images. Most of the cracks were detected inside the resin matrix and at the interface between resin matrix and filler particle. Localized compressive stress induced by sandblasting must have created these surface and subsurface cracks. In particular, the Shofu Block HC blocks presented severe cracks that had propagated along the filler particles. This finding should be related to the several $1-10 \mu \mathrm{m}$ spherical holes detected for the untreated Shofu Block HC. Silane-coupling agents are used to bond the filler particles to the matrix resin. More specifically, the silane-coupling agent $\gamma$-MPS $(\gamma$ - 


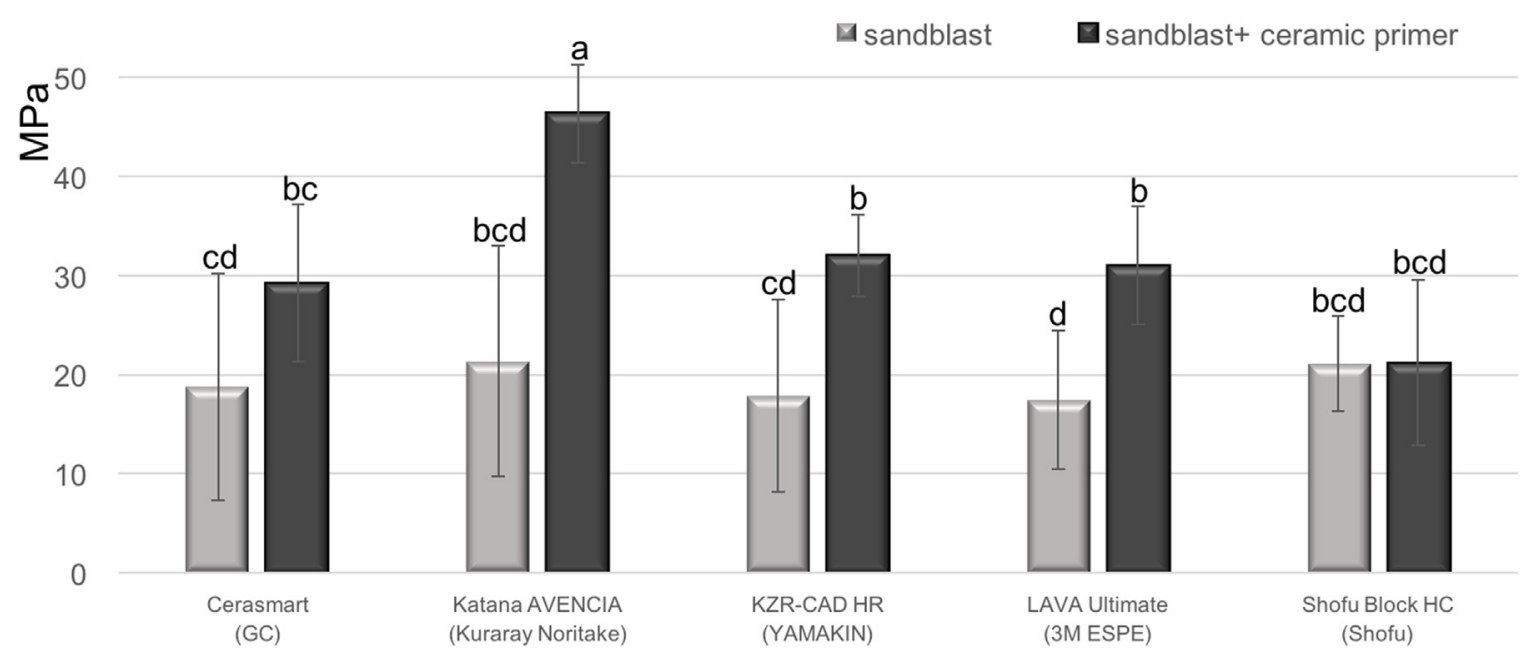

Fig. 7 - Shear bond strength (SBS) of composite cement to the sandblasted composite CAD-CAM blocks that were not ('sandblast') or additionally silane-treated ('sandblast + ceramic primer'). All sandblasted-only CAD-CAM blocks revealed a SBS that did not statistically differ among the composite CAD-CAM blocks. Silane treatment using a separate ceramic primer (Clearfil Ceramic Primer, Kuraray Noritake) significantly increased the SBS of the composite cement to the Katana Avencia, KZR-CAD HR and Lava Ultimate CAD-CAM blocks; although not statistically significant, the SBS to Cerasmart also substantially increased, while that of Shofu Block HC remained nearly the same. The significantly highest SBS was measured when the cement was bonded to the sandblasted and silane-treated Katana Avencia composite block. Different letters indicate statistically significant difference (two-way ANOVA followed by Tukey's multiple comparison test; $\mathrm{p}<0.05$ ).

methacryloxypropyltrimethoxysilane) improves filler-matrix bonding by covalently coating silica with methacrylate double-carbon bonds that can co-polymerize with the resin matrix $[27,28]$. In this way, filler silanization significantly improves the mechanical properties of composite. The morphological findings of this study therefore suggest that the

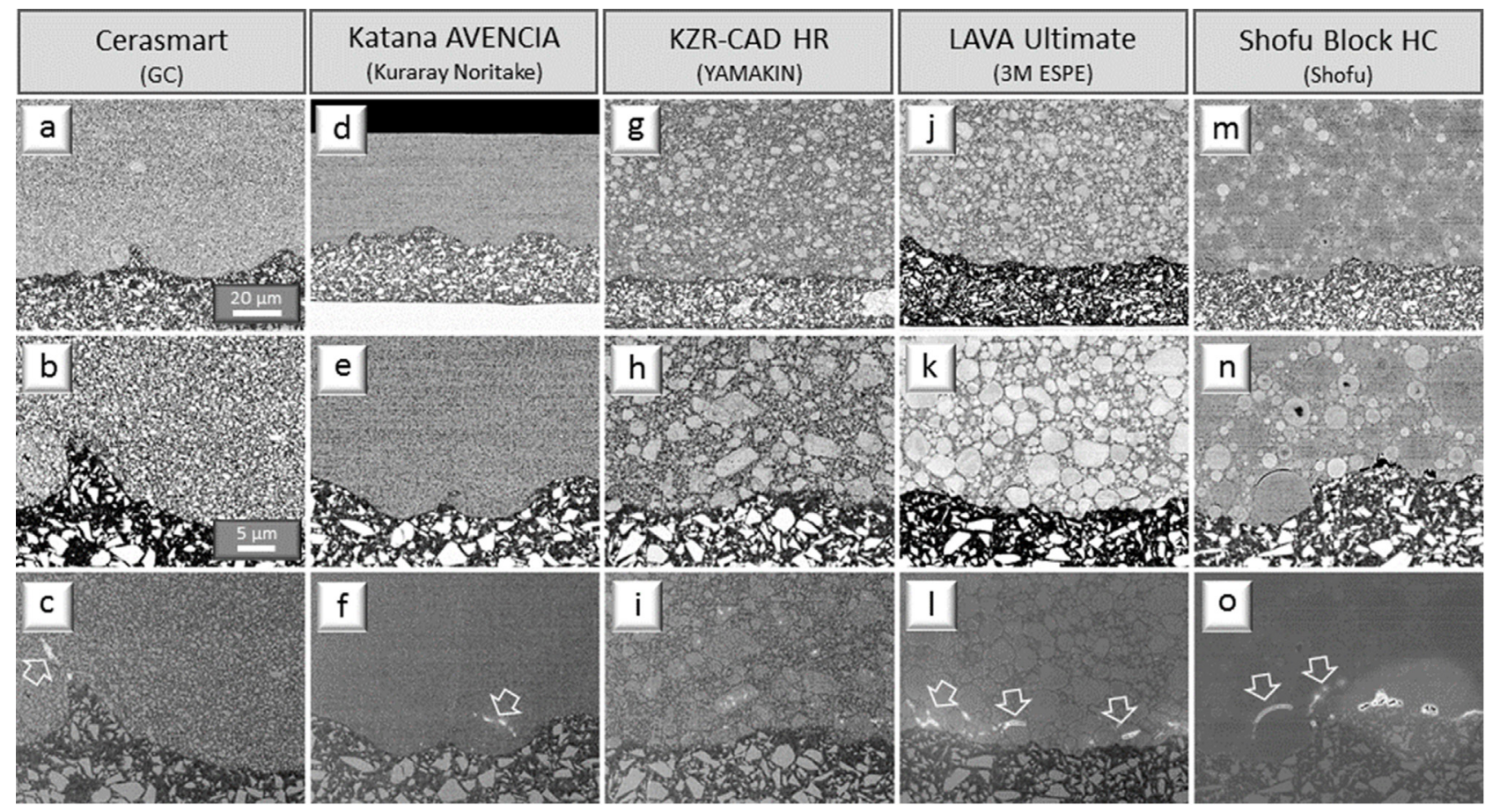

Fig. 8 - Cross-section SEM photomicrographs of the respective cement-composite CAD-CAM block interface. Except Shofu Block HC, all composite CAD-CAM blocks revealed a tight interface with the cement having infiltrated into the roughened sandblasted surface of the composite blocks. However, the cement could not infiltrate into the cracks located deeper in the blocks and more remote from the surface (c,f,l,o: arrows). Shofu Block HC showed several voids at the interface with the cement $(n, 0)$, while the cement had failed to infiltrate into the cracks that had propagated along the filler particles. 

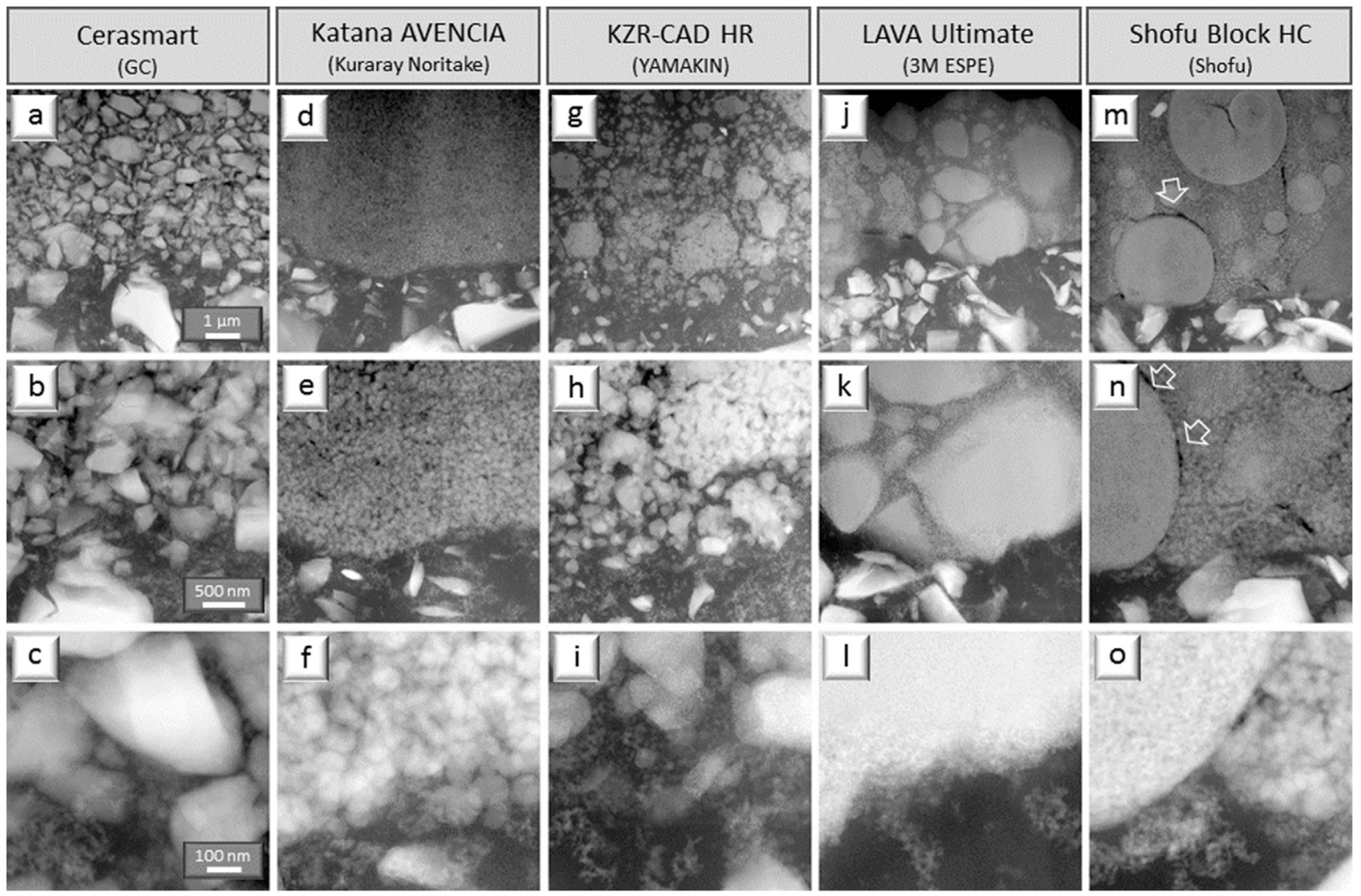

Fig. 9 - Cross-section STEM images of the cement-composite CAD-CAM interface confirmed the tight cement-block interface. Shofu Block HC showed cracks that had propagating along the spherical filler particles (m,n: arrows).

spherical filler particles in Shofu Block HC may not have been adequately silanized.

Although sandblasting affected to different degrees the surfaces of all the composite CAD-CAM blocks tested in this study, sandblasting is obviously also beneficial for luting purposes. For example, sandblasting effectively removes surface contamination, providing a fresh, clean surface that is more receptive for bonding. A study by Yang et al. showed that sandblasting was the most effective method to remove saliva contamination on composite after a restoration tryin simulation [26]. Sandblasting also exposes filler particles. Cross-section SEM images showed that for all the composite CAD-CAM blocks, some filler particles were exposed at the surface by sandblasting. These exposed filler particles so become reachable for silanization. To investigate the effect of silane treatment on bonding performance, the shear bond strength of composite cement to sandblasted composite CAD-CAM blocks with and without prior silanization was measured. Silane treatment significantly increased the bond strength for all the composite CAD-CAM blocks, except for Shofu Block HC. Hence, the null hypothesis that composite CAD-CAM blocks do not benefit from sandblasting and silanization with regard to their bonding receptiveness, should be rejected for all the composite CAD-CAM blocks investigated with the exception of Shofu Block HC. This bond-promoting effect is alike when a micro-filled composite was bonded to a micro-hybrid composite; silane application also improved bond strength [29].
However, the reason why Clearfil Ceramic Primer in this study was unable to improve the bond strength to Shofu Block HC must most likely be attributed due to the latter's severe structural block damage that was before caused by sandblasting. Cross-section SEM and STEM images clearly showed that the cracks had propagated around the filler particles of Shofu Block HC. Furthermore, sandblasting also caused considerable de-bonding of the filler particles, probably in such a way that the subsequent silane coupling prior to luting could not compensate for or overcome the severe block surface and subsurface damage.

Once luted, the resultant cross-section SEM images revealed a tight cement-block interface for all the composite CAD-CAM blocks, again except for Shofu Block HC. Instead, gaps were clearly present at the interface of the cement with the surface of Shofu Block HC. These interfacial gaps may have been caused by air entrapment within the resin matrix, which in turn may have occurred by the severe cracks inside the blocks. Cross-section STEM images further showed filler debonding for Shofu Block HC. Altogether, the cracks around the filler particles inside the CAD-CAM block and the gaps at the interface of the cement with the CAD-CAM block surface did adversely affect the bonding effectiveness of the composite cement to Shofu Block HC.

Composite CAD-CAM blocks are fairly new on the market. As such, they have not been studied much and the most optimal bonding/luting protocol for composite CAD-CAM 
restorations remains also to be explored and established. Most studies investigated 'immediate' bonding effectiveness to composite CAD-CAM blocks [3,30]. Immediate bond strength might primarily result from infiltration/interlocking of the bonding adhesive into the surface irregularities and cracks formed by sandblasting. It has been documented that the sole application of an adhesive, without prior silane treatment, is insufficient [27]; the adhesive was shown not to be capable of chemical bonding to the filler, which caused the bond to degrade over time [31]. Hence, there exists a high need to investigate bonding/luting protocols on their long-term bond durability to composite CAD-CAM blocks.

\section{Conclusion}

Sandblasting composite CAD-CAM blocks produced not only an irregular surface but also surface and sub-surface cracks. This was especially so for Shofu Block HC, the surface and sub-surface of which was severely damaged. Hence, composite CAD-CAM blocks should be sandblasted with reduced pressure. Silane treatment after sandblasting improved bond strength. Combined micro-mechanical and chemical interaction by mild sandblasting followed by silanization appeared a promising bonding/luting protocol for composite CAD-CAM restorations that now needs to be further studied for bond longevity. Such bond-durability studies should be combined with ultra-structural interfacial analysis of composite cements bonded to composite CAD-CAM blocks and exposed to artificial aging through long-term water storage, thermocycling and/or mechanical loading.

\section{Acknowledgement}

This work was supported by JSPS KAKENHI Grant with number JP 16K2045508 and 15K1115807.

\section{REFERENCES}

[1] van Noort R. The future of dental devices is digital. Dent Mater 2012;28:3-12,

http://dx.doi.org/10.1016/j.dental.2011.10.014. Epub 2011 Nov 26. Review.

[2] Miyazaki T, Hotta Y. CAD-CAM systems available for the fabrication of crown and bridge restorations. Aust Dent $J$ 2011;56(Suppl. 1):97-106, http://dx.doi.org/10.1111/j.1834-7819.2010.01300.x.

[3] Lührs AK, Pongprueksa P, De Munck J, Geurtsen W, Van Meerbeek B. Curing mode affects bond strength of adhesively luted composite CAD-CAM restorations to dentin. Dent Mater 2014;30:281-91, http://dx.doi.org/10.1016/j.dental.2013.11.016. Epub 2014 Jan 11.

[4] Ruse ND, Sadoun MJ. Resin-composite blocks for dental CAD-CAM applications. J Dent Res 2014;93:1232-4, http://dx.doi.org/10.1177/0022034514553976. Epub 2014 Oct 24. Review.

[5] Sripetchdanond J, Leevailoj C. Wear of human enamel opposing monolithic zirconia, glass ceramic, and composite resin: an in vitro study. J Prosthet Dent 2014;112:1141-50, http://dx.doi.org/10.1016/j.prosdent.2014.05.006. Epub 2014 Jun 28.

[6] Okada K, Kameya T, Ishino H, Hayakawa T. A novel technique for preparing dental CAD-CAM composite resin blocks using the filler press and monomer infiltration method. Dent Mater J 2014;33:203-9. Epub 2014 Mar 1.

[7] Tsitrou EA, Helvatjoglu-Antoniades M, van Noort R. A preliminary evaluation of the structural integrity and fracture mode of minimally prepared resin bonded CAD-CAM crowns. J Dent 2010;38:16-22, http://dx.doi.org/10.1016/j.jdent.2009.07.003.

[8] Kassem AS, Atta O, El-Mowafy O. Fatigue resistance and microleakage of CAD-CAM ceramic and composite molar crowns. J Prosthodont 2012;21:28-32,

http://dx.doi.org/10.1111/j.1532-849X.2011.00773.x. Epub 2011 Oct 18.

[9] http://www.3m.com/3M/en_US/Dental/Products/Catalog/ ?N=5144788+3294776545\&rt=rud.

[10] Yoshihara K, Nagaoka N, Sonoda A, Maruo Y, Makita Y, Okihara T, Irie M, Yoshida Y, Van Meerbeek B. Effectiveness and stability of silane coupling agent incorporated in 'universal' adhesives. Dent Mater 2016;32:1218-25, http://dx.doi.org/10.1016/j.dental.2016.07.002. Epub $2016 \mathrm{Jul}$ 25.

[11] Inokoshi M, De Munck J, Minakuchi S, Van Meerbeek B. Meta-analysis of bonding effectiveness to zirconia ceramics. J Dent Res 2014;93(4):329-34, http://dx.doi.org/10.1177/0022034514524228. Epub 2014 Feb 21.

[12] da Costa TR, Serrano AM, Atman AP, Loguercio AD, Reis A. Durability of composite repair using different surface treatments. J Dent 2012;40:513-21,

http://dx.doi.org/10.1016/j.jdent.2012.03.001.

[13] Suliman AH, Swift EJ, Perdigao J. Effects of surface treatment and bonding agents on bond strength of composite resin to porcelain. J Prosthet Dent 1993;70:118-20.

[14] Zhang Y, Lawn BR, Rekow ED, Thompson VP. Effect of sandblasting on the long-term performance of dental ceramics. J Biomed Mater Res B Appl Biomater 2004;71:381-6, http://dx.doi.org/10.1002/jbm.b.30097.

[15] Brosh T, Pilo R, Bichacho N, Blutstein R. Effect of combinations of surface treatments and bonding agents on the bond strength of repaired composites. J Prosthet Dent 1997;77:122-6.

[16] Ozcan M, Barbosa SH, Melo RM, Galhano GA, Bottino MA. Effect of surface conditioning methods on the microtensile bond strength of resin composite to composite after aging conditions. Dent Mater 2007;23:1276-82, http://dx.doi.org/10.1016/j.dental.2006.11.007.

[17] Rodrigues Jr SA, Ferracane JL, Della Bona A. Influence of surface treatments on the bond strength of repaired resin composite restorative materials. Dent Mater 2009;25:442-51, http://dx.doi.org/10.1016/j.dental.2008.09.009.

[18] Loomans BA, Cardoso MV, Roeters FJ, Opdam NJ, De Munck J, Huysmans MC, et al. Is there one optimal repair technique for all composites? Dent Mater 2011;27:701-9, http://dx.doi.org/10.1016/j.dental.2011.03.013.

[19] Peumans M, Valjakova EB, De Munck J, Mishevska CB, Van Meerbeek B. Bonding effectiveness of luting composites to different CAD/CAM materials. J Adhes Dent 2016;18(4):289-302.

[20] Lise DP, Van Ende A, De Munck J, Vieira L, Baratieri LN, Van Meerbeek B. Microtensile bond strength of composite cement to novel CAD/CAM materials as a function of surface treatment and aging. Oper Dent 2016;30(September). Epub ahead of print.

[21] http://www.gcamerica.com/lab/products/CERASMART/ CERASMART_12IFU.pdf for Cerasmart (GC). 
[22] http://multimedia.3m.com/mws/media/7473920/lava-ultimate -restorative-instructions-for-use-english.pdf for Lava Ultimate (3M ESPE).

[23] http://www.shofu.de/wp-content/uploads/2016/03/BPZ_SHOFU_ Block_HC_2014-09AXDEESFRGBITLTNLPLRORU.pdf for Shofu Block HC (Shofu).

[24] http://www.kuraraynoritake.jp/product/cad_ref/pdf/ avenciablock_howto.pdf for Katana Avencia (Kuraray Noritake) in Japanese.

[25] http://www.yamakin-gold.co.jp/technical_support/webrequest/ pdf/kzr-cad_hrb.pdf for KZR-CAD-HR (Yamakin)in Japanese.

[26] Yang B, Wolfart S, Scharnberg M, Ludwig K, Adelung R, Kern M. Influence of contamination on zirconia ceramic bonding. J Dent Res 2007;86:749-53.

[27] Yoshida Y, Shirai K, Nakayama Y, Itoh M, Okazaki M, Shintani H, et al. Improved filler-matrix coupling in resin composites. J Dent Res 2002;81:270-3.
[28] Spitznagel FA, Horvath SD, Guess PC, Blatz MB. Resin bond to indirect composite and new ceramic/polymer materials: a review of the literature. J Esthet Restor Dent 2014;26:382-93, http://dx.doi.org/10.1111/jerd.12100. Epub 2014 Apr 23.

[29] Soares CJ, Giannini M, Oliveira MT, Paulillo LA, Martins LR. Effect of surface treatments of laboratory-fabricated composites on the microtensile bond strength to a luting resin cement. J Appl Oral Sci 2004;12:45-50.

[30] Stawarczyk B, Krawczuk A, Ilie N. Tensile bond strength of resin composite repair in vitro using different surface preparation conditionings to an aged CAD-CAM resin nanoceramic. Clin Oral Invest 2015;19:299-308, http://dx.doi.org/10.1007/s00784-014-1269-3. Epub 2014 Jun 15.

[31] Yoshida Y, Shirai K, Nakayama Y, Itoh M, Okazaki M, Shintani $\mathrm{H}$, et al. Improved filler-matrix coupling in resin composites. J Dent Res 2002;81:270-3. 